\title{
La metáfora computacional y la delimitación al ámbito representativo en las ciencias cognitivas
}

\author{
Juan A. Moreno \\ Universidad de Málaga
}

The comparison of the human body with the machine is an ancient matter and it has contained more attention from the Cartesian Physics for its dualism mind-body. In this article, we analyze the historical process for which the cognitive Philosophers have finished using methodically the computer as model for their comprehension of the human mind. Also we study how they have realized their methodical delimiting to the representative area, and the cognitive earnings that both methods have provided to them.

Las ciencias cognitivas son las ciencias que estudian la cognición.' En un sentido poco elaborado "cognición" significa "conocimiento": tomar cuenta de una realidad dada, recepción de información. En un sentido más elaborado significa uso y manejo de conocimiento, o lo que es lo mismo: manipulación de la información. La diferencia entre recibir y elaborar información es la misma que encontramos entre la percepción de un calor sofocante y la decisión de darse una ducha fría para aliviar el calor. Según la primera definición ambos objetos serían cognición. Según el sentido elaborado, el primer caso correspondería a información y sólo en el segundo caso habría cognición propiamente. El sujeto ha acudido al conocimiento que tiene de la ducha fría y de su eficacia para aliviar el calor. Tanto los procesos de simple conocimiento, como los procesos propiamente cognitivos son procesos mentales. La mente actúa en todos ellos, pero en los procesos cognitivos se implica más. En palabras de

${ }^{1}$ Estos dos sentidos del significado de cognición los tomo del artículo del prof. Pascual MARTínez: "Procesos mentales y cognitivismo", Revista de Filosofia V-7 (1992), pp. 155-6. 
Martínez Freire: "Sentir placer fisico es menos mental que demostrar un teorema matemático"2. La manipulación de la información es una tarea común de la mente humana, animal o artificial. Para un cognitivista, "la mente no es un privilegio humano sino que es legítimo hablar de mentes animales e incluso de mentes mecánicas". 3

\section{El uso metódico de la metáfora computacional}

La comparación del cuerpo humano con la máquina es asunto antiguo y cobra la mayor atención desde la física cartesiana ${ }^{4}$ precedente del dualismo moderno, mente-cuerpo. La ciencia del siglo XVII se divide, en relación con su concepción antropológica y fisica en mecanicistas (Descartes, Newton) y dinamicistas como Leibniz. En la segunda mitad del siglo $X X$, ¿cuál ha sido el proceso histórico por el que los cognitivistas han acabado usando metódicamente el ordenador como modelo para la comprensión de la mente humana?

McCulloch, Pitts, Neumann y Wiener. En 1943, Warien McCulloch y Walter Pitts demostraron que las operaciones de una célula nerviosa y sus conexiones con el resto de las células nerviosas podrian ser descritas perfectamente por modelos lógicos. Los nervios eran equiparables a enunciados lógicos, y su propiedad de ser activado o no era comparable a las operaciones de cálculo

2 P. MARTINEZ: "Procesos mentales...", p. 155.

3 Pascual Martínez: "El impacto de las ciencias cognitivas en la filosofĩa del conocimiento", Philosophica Malacitana, sup. 3, 1995, p. 58

${ }^{4}$ Los modelos mecanicistas que desembocan en la máquina de Turing, comienzan históricamente en la física cartesiana y en D'Holbach, Hobbes, Spinoza y sobre todo La Mettrie. Más tarde aparecerán los modelos de los socialistas utópicos y de los pre-revolucionarios que acabarán concurriendo con los desarrollos logicistas que partieron de Leibniz, para pasar al progreso actual de la lógica y la cibemética. La diferencia especifica del hombre frente a la máquina es deféndida no sólo por filósofos realistas como Mariano ARTIGAs (La inteligibilidad de la naturaleza, Pamplona: Eunsa 1992, pp. 467 ss.), sino por físicos como Roger PENROSE (La nueva mente del emperador, Madrid: Mondadori 1991), filósofos de la ciencia como John EcCles y Karl Popper (El Yo y su cerebro, Barcelona: Paidos 1985), biólogos como Christopher WILLS (El cerebro fugitivo, Barcelona: Paidos 1994), y físicos como el Premio Nóbel (1969) Murria Gell-ManN (El Quark y el jaguar; Barcelona: Tusquets 1994). 
proposicional. Así como Turing demostró que podria haber potentes computadoras pensantes, McCulloch y Pitts mostraron que había al menos una potente máquina - el cerebro-cuyas operaciones eran equiparables con los principios de la lógica.

Aquel mismo año, en Princeton, Neumann, tuvo ocasión de conocer los innovadores trabajos de McCulloch, Pitts y Turing. Alli elaboró lo que más tarde se conoció como "programa almacenado". La idea del "programa almacenado" era sencillamente crear un programa que instruyese a la máquina de Turing. La computadora podría controlarse mediante un programa almacenado en su memoria interna, de modo que no tuviera que ser laboriosamente reprogramada para cada nueva tarea. Cinco años más tarde, en 1948, Neumann sintetizó todas sus ideas sobre los ordenadores en su libro Cibernética, donde concluye que cibernética es todo el campo de la teoría del control y la comunicación, ya se trate de la máquina o del animal.

Por su parte, Wiener, que también estaba al tanto de los trabajos de McCulloch y Turing, descubrió una coincidencia entre los sistemas de conexiones lógicas y el sistema nervioso. En 1943 publicó "Conducta, propósito y teleología", un artículo en que defendía que si una máquina posee retroalimentación, es legítimo decir que posee "metas", y si es capaz de calcular las diferencias entre esas metas y su desempeño efectivo, puede luego reducir tales diferencias. Para Wiener - por tanto- las máquinas estaban dotadas de propósitos, igual que la mente humana.

Así comienza el uso metódico de la computadora como metáfora del cerebro. De ahí en adelante, los sucesivos avances en los estudios neurológicos y en la inteligencia artificial han ido demostrando más la pertinencia de este método. Sobre todo porque tanto el ordenador como la mente humana tienen conocimientos, y para que haya conocimiento tiene que haber computación y algoritmos computacionales. El estudio de la computación y de los algoritmos que la permiten da luces para entender mejor el funcionamiento de la mente humana y artificial. 
La computación en el conocimiento. La mayoría de los cognitivistas consideran que un ordenador programado adecuadamente puede pensar y tener otros estados cognitivos; es decir, para ellos el ordenador programado es una mente. Algunos cognitivistas $^{5}$-más prudentes- piensan que los ordenadores son solamente poderosos instrumentos para el estudio de la mente humana, permitiéndonos formular y comprobar las hipótesis de un modo riguroso y preciso. Ambos grupos de cognitivistas están de acuerdo en el paralelismo entre la inteligencia artificial y la inteligencia humana, pero disienten respecto al alcance de este paralelismo. Obviamente, en la mente humana se dan procesos "espirituales" no compartidos por las otras mentes (autoconciencia, proyecciones vitales, voliciones indeterminadas...). Esto significa que en el hombre existe un ámbito no físico.

Pero lo que aquí nos interesa es que en toda mente (humana, animal o artificial) los procesos mentales se identifican con procesos de computación. Esto que parece obvio en los ordenadores, supuso una revolución en el campo de la psicología cuando se descubrió en la mente humana. Fue la psicología cognitiva quien postuló en los años sesenta que el pensamiento humano èra computacional. Entonces los psicólogos hubieron de pedir ayuda a la inteligencia artificial para conseguir conceptos adecuados a la realidad descubierta. El paradigma que había asumido por entonces la inteligencia artificial fue el de la computación clásica, también llamada de von Neumann. La computación clásica consiste en la manipulación serial o secuencial de símbolos (representaciones ${ }^{6}$ ) definida en términos de reglas formales (más o menos fijas). A mediados de los años ochenta, se fue imponiendo el paradigma de computación conexionista. Es a partir de entonces cuando muchos psicólogos cognitivistas tomaron este nuevo modelo. La computación conexionista se diseña como manipulación de unidades

5 Esta distinción la ha hecho John Searle denominando a la primera postura: "inteligencia artificial fuerte" y a la segunda: "inteligencia artificial débil o prudente". Aunque Searle está a favor de la opción débil, casi todos los cognitivistas están a favor de la existencia de "mentes mecánicas". Cf. John SEARLE: "Minds, Brains and Programs", The Behavioral and Brain Sciences 3 (1980), pp. 417-24.

${ }^{6}$ Los instrumentos de la computación son las representaciones mentales. 
simples con cierto grado de activación y que están máximamente interconectadas, de tal manera que se produce entre las unidades excitaciones e inhibiciones, $y$ además tal computación sigue un procesamiento paralelo, no secuencial.

En la actualidad los psicólogos cognitivistas parecen divididos entre ambos modelos (clásico y conexionista); de todos modos no cabe duda de que el modelo conexionista, con sus numerosas interconexiones entre neuronas artificiales, se asemeja mucho más al cerebro humano.

Los algoritmos en el conocimiento. Un algoritmo es un conjunto de instrucciones para operar. Un algoritmo es un procedimiento mecánico, sometido a reglas fijas y definidas, que permite resolver un problema o ejecutar una tarea en un número finito de pasos. Cuando se programa un ordenador, deben estar explícitos tanto los algoritmos como las ocasiones en que se han de emplear. Estas ocasiones no se pueden reducir a un sólo caso, sino a una clase de casos. Esta sería la definición ideal de algoritmos, lo que Martínez Freire" denomina el algoritmo "fuerte". Sin embargo es mucho más interesante y eficaz el denominado algoritmo "débil" o método heurístico. Muchos algoritmos ideales son ambiguos, con lo cual, y según los casos de aplicación, necesitarán ciertas reglas o instrucciones suplementarias para poder ser desarrollados. Gracias también a los medios heurísticos, un algoritmo prediseñado para una actividad, puede ser transferido a otra actividad en la que también tiene éxito. Pues bien, el funcionamiento de estos algoritmos débiles hace que cada vez más los ordenadores se asernejen a la mente humana. Los programadores van a ir incluy'endo progresivamente estrategias de búsqueda de soluciones, más que algoritmos cerrados o fuertes.

Las criticas al uso metódico de la metáfora computacional: dentro de los científicos, también han aparecido duras críticas al uso de esta metáfora como método de investigación. Podemos descubrir dos tipos de criticas.

${ }^{7}$ P. MARTÍNEZ: “El impacto...”, p. 60. 
Por una parte, hay quienes piensan que los ordenadores no son sino el último de una larga serie de modelos inadecuados de la cognición humana. ${ }^{8}$ Estos no encuentran razón alguna para pensar que el modelo de la máquina actual tenga mejor destino. Estos criticos consideran un gran error concebir a los organismos activos como sistemas de procesamiento de la información.

Entre los que comparten esta opinión tenemos a muchos antropólogos, para quienes la clave del pensamiento radica en gran. medida en fuerzas históricas y culturales que se hallan fuera de la cabeza del hombre. Estas fuerzas no se pueden representar en los ordenadores debido a que un ordenador carece de historia, de tradición y de cultura. ${ }^{9}$ Por esta razón el modelo computacional será siempre deficiente.

Por otra parte, están quienes consideran a los ordenadores como meros juguetes que interfieren más que ayudan al estudio del pensamiento humano. Sostienen que el hecho de que se pueda simular gran parte del comportamiento de la mente humana, distrae la atención respecto a un estudio detenido $y$ realista del comportamiento de la mente humana. Esta opinión la comparten algunos neurólogos que piensan que el mismo cerebro dará las respuestas planteadas sobre su funcionamiento, sin necesidad de acudir a un modelo externo.

Por lo general, el grado en que una disciplina está próxima a la ciencia cognitiva puede medirse por el grado en que está ligada a las computadoras. Entre las disciplinas que se han manifestado más escépticas respecto al modelo computacional se encuentran la psicología y la lingüistica. También la filosofia ha elaborado críticas a la pertinencia de la metáfora computacional como método.

\footnotetext{
${ }^{8}$ Como modelo le preceden el tablero de conmutador, la bomba hidráulica o el holograma.

${ }^{9}$ Cuando un nuevo ordenador surge en el mercado, no se encuentra inmerso en ninguna tradición cultural, carece de prejuicios históricos.
} 


\section{La delimitación al ámbito representativo como método}

Los estados internos de la mente: Otra de las características de las ciencias cognitivas es su delimitación metódica y temática al ámbito representativo. Aunque algunos científicos cognitivistas no realizan esta delimitación. Los cognitivistas entienden por ámbito representativo o de representación lo que Hegel denominó objetividad, lo que William James llamó conciencia, lo que Husserl denominó intencionalidad. Representativo es hacer presente una cosa.

La raiz de esta delimitación la encontramos a reacción contra el conductismo con la que nacieron las ciencias cognitivas. Vale la pena recordar que para el conductismo no existian procesos mentales ni una "vida mental", sino que los fenómenos psicológicos se reducian a la conducta observable y registrable pública y externamente. Las ciencias cognitivas reaccionaron afirmando que la mente (humana, animal ${ }^{10}$ o artificial) es un sistema de estados internos (procesos mentales) que representa (con diferentes grados de convencionalidad y eficacia) el mundo externo. La mente es el conjunto de diversas clases de representaciones. Para los cognitivistas existen procesos mentales internos que pueden no manifestarse en la conducta. En cuanto sistema de estados internos, la mente constituye un conjunto organizado, una estructura en la que deben distinguirse aspectos preformados asi como aspectos desarrollados, inducidos o aprendidos.

Más tarde, Claude Shannon, ingeniero electricista, observó en los años cuarenta que podian utilizarse los principios de la lógica (en lo que respecta a la verdad o falsedad de las proposiciones) para describir los dos estados (abierto y cerrado; encendido y apagado) de las llaves relés electromecánicas. Por esta modificación binaria, los circuitos eléctricos podían abarcar operaciones fundamentales del pensamiento. Su Teoría de la Información consistia en que puede

\footnotetext{
${ }^{10}$ Según el profesor Martínez (cf. P. Martínez: "El impacto...”, p. 60), después del libro de Donald GrIfFIn: Animal Minds, Chicago: The University of Chicago Press 1992, ya no se debería dudar de que la mente humana es comparable con la mente animal.
} 
considerarse a esta como totalmente independiente del contenido o materia, teniendo sólo en cuenta que es una admisión singular entre dos alternativas igualmente admisibles. La cantidad de información mínima para elegir entre una información $u$ otra es el bit (binary digit). Por ejemplo, para elegir un mensaje entre ocho alternativas distintas bastan tres bits. La principal aportación de la teoría de la información de Shannon consiste en que consigue desligar la información de toda materialidad. La información ni es materia ni es energía, es una representación significativa.

No todos los cognitivistas conceptualizan del mismo modo las representaciones mentales. Algunos opinan que sólo hay una forma de representación mental (la que incluye proposiciones 0 enunciados). Otros piensan que hay dos formas: una semejante a figuras e imágenes, y otra más próxima a las proposiciones. También hay quienes creen posible postular múltiples formas, y consideran imposible determinar cuál de ellas es la correcta.

La mente, para las ciencias cognitivas no es una sustancia aislada, sino que es el conjunto de diversos procesos mentales y representaciones del mundo externo. Por mundo externo se entiende todo lo real que está más allá de la vida mental. Aquí entra en juego un concepto muy usado en la gnoseología: la intencionalidad. La intencionalidad significa el acto de un conocimiento de remitir a algo. Todo conocimiento de algo es intencional. Ahora bien, desde el punto de vista de las ciencias cognitivas, la intencionalidad es interpretada con una claridad nueva: es la capacidad de representar. Todo estado mental interno es una representación que remite a algo externo, por tanto, todo estado mental es intencional. Como los estados internos son comunes a los animales y a las máquinas, muchos cognitivistas afirman que también los animales y las máquinas poseen intencionalidad.

Es indudable que existen estados internos o mentales en las máquinas si sólo atendemos a la instancia física de dichos estados, pues toda representación tiene un componente fisico. Ahora bien, el problema surge cuando tenemos en cuenta que toda representación tiene además un valor semántico. Los estados internos de la máquina 
¿tienen valor semántico en sí? Para responder a esta pregunta podemos atender a la hipótesis de la representación del conocimiento de Brian Smith." Según su hipótesis, cualquier proceso inteligente realizado mediante un mecanismo estará compuesto de ingredientes estructurales. Estos ingredientes estructurales tienen dos funciones independi $:$ ntes entre sí, a saber: en primer lugar, nosotros -como observadores externos - interpretaremos esas estructuras como represeniaciones de una o varias explicaciones proposicionales del conocimiento que el proceso global exhibe. Dicho más claro: nosotros asignamos un valor semántico al estado estructural interno que le es extrínseco al ordenador. En segundo lugar, esos ingredientes estructurales juegan un papel formal-causal en la producción de la conducta que manifiesta ese conocimiento. Por tanto las representaciones de la máquina tienen una función semántica externa que se la da el programador y el usuario, $\mathrm{v}$ tienen también una función interna que transforma la conducta del ordenador.

Los ordenadores exhiben conocimientos y poseen representaciones. Sus representaciones influyen formal y causalmente en ellos. ¿Podrán influir alguna vez por -su valor semántico? ¿Podrán las máquinas alguna vez interpretar algunos de sus estados internos?

Las dos dimensiones de la delimitación al ámbito representativo. Esta delimitación se realiza hacia arriba y hacia abajo. Hacia arriba, los cognitivistas dejan fuera el estudio del contexto cultural, sociológico e histórico. De este modo se gana en precisión. Hacia abajo, subordinan los conocimientos particulares científicos (sobre neuronas, microprocesadores...) al ámbito representativo. Así se evita el materialismo. Explico mejor ambas delimitaciones en el siguiente esquema por niveles:

11 Brian SMrTh: Reflections and Semantics in a Procedural Language, Cambridge: Tech. Report MIT/LCS/TR-27, 1982. 


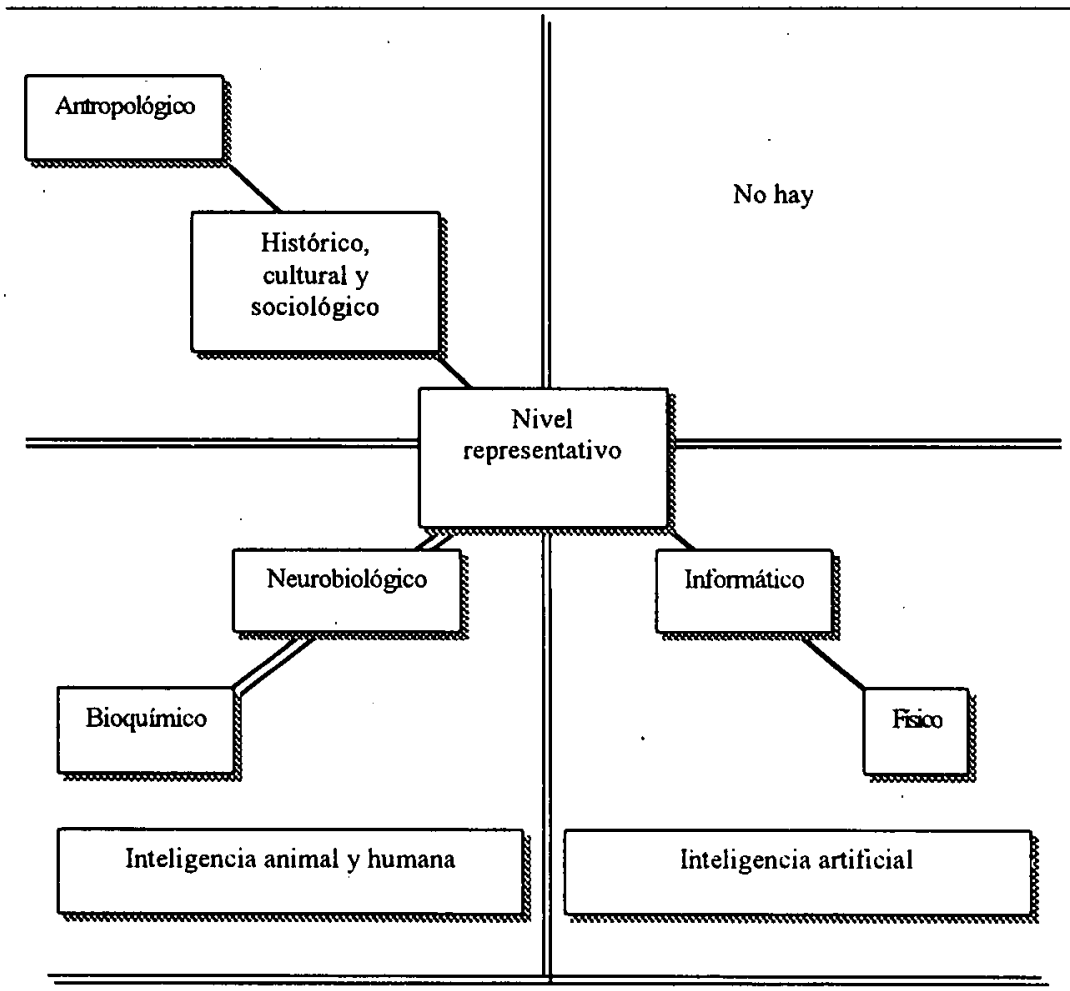

La delimitación y exclusión del nivel cultural-sociológico con respecto al ámbito representativo se realiza por dos razones. La primera es: los factores culturales y emocionales, si bien pueden ser importantes para el funcionamiento cognitivo, complicarian innecesariamente los estudios científicos en estos momentos. Las ciencias cognitivas serian ahora impracticables si se quisiera tener en cuenta todos estos fenómenos individualizadores. Más que obviar estos factores, se posponen indefinidamente. La otra razón es: lo cultural y lo afectivo no son factores lógicos ni predecibles, por lo que harian peligrar la precisión de los estudios de las representaciones mentales. En la actualidad, la corriente principal de científicos cognitivistas no tiene animadversión contra la esfera de los afectos, o contra el contexto que circunda cualquier acción o pensamiento, o contra los análisis culturales o históricos. Sin 
embargo, en la práctica, intentan deslindar estos elementos todo lo posible de su investigación.

La delimitación al ámbito representativo nos libra del relativismo, porque integra los conocimientos sobre la sociología, la historia y la cultura, ignorando la influencia irracional que estos puedan tener en el contenido del ámbito representativo. "Da igual las causas de porqué razonamos así (métodos) y porqué perseguimos esos fines cognocitivos, el hecho es que razonamos así, y tenemos éxito". Esta influencia irracional provendria de las razones no científicas (personales) de los científicos. ¿Qué más da que Einstein pretendiese hacer una teoría original para alcanzar la fama? ¿Qué más da que su teoria hubiese podido ser promocionada políticamente por los judios? Mentalménte está bien construida y su aplicación proporciona resultados prácticos evidentes.

Esta delimitación ha encontrado sus críticas. Estas son de dos tipos: críticas internas (desde las ciencias cognitivas), y críticas externas que son más destructivas. Las críticas internas dicen que los factores "externos" son esenciales en la experiencia humana. Estos factores pueden y deben ser estudiados por las ciencias cognitivas en la medida de lo posible. Asi se evitarian unas ciencias cognitivas antisépticas, que ponen entre paréntesis estas dimensiones personales de modo artificial. Ellos sostienen que los cientificos deberian trabajar con ahínco para incorporar cabalmente dichas dimensiones en sus modelos del pensamiento y la conducta. Otra critica interna afirma que las representaciones (propósitos, creencias, intenciones...) son usadas necesariamente por el lenguaje común. Pero no es necesario que haya un lenguaje científico y un nivel de análisis separados de su representación mental. Desde este punto de vista, sería posible pasar de forma directa de los planes de la mente al sistema nervioso, ya que es aqui, en última instancia, donde todos los planes o propósitos deben estar representados.

Las criticas externas sostienen que factores como los afectos, el contexto o la historia nunca serán explicables por las ciencias naturales. Ellas son temas de las ciencias humanísticas o estéticas. Como estos factores son centrales para la experiencia humana, 
cualquier ciencia que los excluya está de entrada condenada al fracaso.

La segunda delimitación al ámbito representativo se realiza excluyendo los conocimientos neurobiológicos, informáticos, fisicos y bioquimicos. En esta segunda delimitación también hay desacuerdos. Algunos consideran que lo mejor es que las ciencias cognitivas sigan su curso sin preocuparse de tener un conocimiento minucioso del sistema nervioso. No creen que los modernos descubrimientos neurológicos sean vitales para el progreso de las ciencias cognitivas. Las razones que estos aducen son principalmente dos: a) aún no se ha logrado un conocimiento suficiente sobre la actividad cerebral; b) sólo investigando al margen de la actividad neuronal, podemos. asegurar la independencia del nivel representativo. A medida que el nivel cognitivo se afiance y se hagan mayores descubrimientos en las ciencias del cerebro, es posible que se reduzca esta distancia autoimpuesta. De todos modos, la gran mayoria de los cognitivistas no apoyan esta segunda delimitación y abogan por el conocimiento de los niveles inferiores.

Si los problemas del pensar se resuelven desde el nivel representativo; desde él podremos explicar todos los demás niveles que entran a jugar en la cognición. En el gráfico anterior, el ámbito representativo está en medio, y hacia abajo encontramos en primer lugar el nivel neurobiológico (caso de la mente humana y animal) y el nivel informático (caso de la inteligencia artificial), y a los pies están el nivel bioquímico (hombre y animal) y el nivel físico (inteligencia artificial) que son los infimos.

Si nos damos cuenta, este método de explicación de arriba hacia abajo evita los reduccionismos materialistas. En la línea biológica se evita que la neurobiología se explique desde la quimica y que el pensamiento se explique desde la neurobiologia. Del mismo modo, en la inteligencia artificial este método evita que la tecnologia informática se explique desde la fisica, y que el pensamiento de la máquina se explique desde la tecnología informática. 
Hilary Putnam ha afirmado que las operaciones lógicas llevadas a cabo por el hombre podrian explicarse al margen del cerebro, del mismo modo que el software puede explicarse al margen del hardware. Putnam ${ }^{12}$ hace una observación obvia: dos ordenadores con configuraciones distintas (distinto hardware), al ser programados por un programa similar (mismo software), pueden realizar operaciones similares. Por otra parte, dos ordenadores con la misma configuración, realizarán operaciones totalmente distintas si se los programa de modo distinto. De aquí podemos deducir tres conclusiones:

Existe una cierta independencia del software con respecto al hardware. Recordemos que la descripción lógica de la máquina de Turing no incluía especificaciones referidas a su materialización fisica:

El ámbito representativo (los estados mentales) es independiente de su soporte material. Esta independencia ilustra la independencia que existe en el hombre entre la operatividad mental (ideas) y la configuración física (química o eléctrica) del cerebro.

El comportamiento cognoscitivo de un sistema $u$ organismo se define ante todo por su organización funcional, siendo de importancia secundaria la constitución fisica del sistema. En pacientes donde ha sido totalmente dañado la parte del cerebro dedicada a alguna función concreta (el lenguaje, por ejemplo) al cabo de un tiempo, comienzan a usar otras zonas del cerebro para seguir hablando. Lo espiritual ordena hacia si lo material.

Las ciencias cognitivas han logrado demostrar algo que la filosofia habia intuido hacía tiempo: el pensamiento tiene su propio campo de independencia con respecto al cuerpo: no está predeterminado por

12 "Un espíritu incorpóreo podría presentar un cierto programa, un cerebro podría presentar un cierto programa, una máquina podría presentar un cierto programa, pero la organización funcional de los tres (el espiritu incorpóreo, el cerebro y la máquina) podría ser exactamente la misma aun cuando su materia y su sustancia tuesen completamente diferentes" (Hilary PuTnAM: Razón, verdad e Historia, Madrid: Tecnos 1967, p. 86). 
éste. Sólo cabe hablar de predeterminación cuando admitimos algún tipo de materialismo; si decimos que lo menos determina lo más. Ahora bien, decir que lo más informa lo menos no es determinista: Lo que se está diciendo es que el pensar ordena lo que le es inferior: En terminología aristotélica, lo neuronal seria la causa eficiente que eleva y ordena lo bioquímico (causa material) al orden de lo mental (causa final).

Podemos conocer qué nos dice la física, la biología, la informática, etc... sin que tengamos que depender de sus métodos y de sus extrapolaciones no pertinentes. "La mente tiene como condiciones al organismo y al medio, pero no es el organismo ni el medio... no se reduce ni a los principios de la fisiologia ni al orden impuesto por el mundo externo", 13 y por tanto sus leyes son distintas de las leyes de éstos. La mente establece fines y medios propios (el conocimiento) distintos de los fines del mundo físico o neuronal. Esto también se ha llamado "el modelo funcionalista de la mente". Así se da un paso sobre: los conductistas, quienes eliminaban la conciencia o la limitaban a ser mera administradora del arco reflejo.

\section{Conclusión: los resultados de estos métodos:}

El ámbito representativo puede incluir de modo objetivo ${ }^{14}$ todos los demás niveles de arriba y de abajo. La delimitación al ámbito representativo no sólo aleja el peligro materialista. También elimina el riesgo de perder rigor cientifico al querer hacer depender las ideas de instancias supra-individuales: nivel sociológico y cultural. Ellos no niegan que exista una base biológica en el pensar, ni tampoco una supeditación del pensar a niveles supra-individuales. Pero defienden la autonomia temática del nivel representativo para hacer una ciencia que permita la interdisciplinariedad (a fuerza de no ser materialista) sin perder el rigor científico (a fuerza de no convertirse en antropología cultural o sociología).

13 Alicia Rodriguez: "Posiciones relevantes en el tema de la conciencia", Philosophica Malacitana, sup. 3 (1995), p. 149.

14 "De modo objetivo" significa que el ámbito representativo no es perturbado por esos niveles, 'sencillamente los conocimientos de esos niveles son objetivados; conocidos y registrados en el ámbito representativo. 
Esta limitación al ámbito representativo es lo que nos abre la posibilidad de usar metódicamente la metáfora computacional: ${ }^{15} \mathrm{La}$ mente del ordenador también tiene fines y medios independientes de las leyes fisicas de sus circuitos integrados. No cabe el materialismo. $\mathrm{Si}$ alguien argumentara: los fines y los medios no son del ordenador, sino de los programadores o usuarios, habria que contestarle: "Estás considerando el ámbito de los intereses irracionales-personales ajenos al mundo de la lógica interna". Se puede aislar el ámbito representativo del ordenador de las causas externas superiores (programador $\mathrm{y}$ usuario) $\mathrm{y}$ de las causas externas inferiores (hardware y fisica). El ámbito representativo permite enunciados verdaderos con aspiración de universalidad. Este me parece que es uno de los resultados más interesantes de las ciencias cognitivas.

${ }^{15}$ Así lo hace. Jerry FoDor (La modularidad de la mente, Madrid: Morata 1986, $\mathrm{p}$. 67) y JOHNSON:-2ARD (The computer and the Mind: An introducction to Cognitive Science, Cambr iuge: Harvard University Prese 19\%" ${ }^{2}$, pp. 25-6). 
Copyright of Tópicos. Revista de Filosofía is the property of Universidad Panamericana and its content may not be copied or emailed to multiple sites or posted to a listserv without the copyright holder's express written permission. However, users may print, download, or email articles for individual use. 